\title{
Galactic structure studies with SCUSS and SDSS surveys
}

\author{
Cuihua $\mathrm{Du}^{1}$ and Xiyan Peng ${ }^{1,2}$ \\ ${ }^{1}$ College of Physical Sciences, University of the Chinese Academy of Sciences, \\ Beijing 100049, China \\ email: ducuihua@ucas.ac.cn \\ ${ }^{2}$ National Astronomical Observatories, Chinese Academy of Sciences, Beijing 100012, China
}

\begin{abstract}
Based on the South Galactic Cap U-band Sky Survey (SCUSS) and SDSS observations, we adopted the star-count method to analyze the stellar distribution in different directions of the Galaxy. We find that the scale height of the disk may be variable with the observed direction, which cannot simply be attributed to statistical errors. The main reason can be possibly attributed to the disk (mainly the thick disk) being flared, with a scale height increasing with radius. The axis ratio of the Galactic halo is in the range $0.4-0.6$. This finding supports Galactic models with a flattened inner halo, partly formed through a merger early in the Galaxy's history.
\end{abstract}

Keywords. Galaxy: fundamental parameters, Galaxy: halo, Galaxy: stellar component, Galaxy: structure

The star-count analysis has provided a picture of the basic structural and stellar populations of the Galaxy. Up to now, the basic stellar components of the Milky Way are the thin disk, thick disk, stellar halo and central bulge, albeit that the inter-relationships and distinction amongst different components remain subject to some debate. In this study, we use the South Galactic Cap U-band Sky Survey (SCUSS) and SDSS observations to study the Galactic structure. We will report on our investigation of star counts, extending our analysis to include different directions within the Galaxy. The fields used in this paper are towards the Galactic centre and the anticentre direction $\left(0^{\circ}<l<180^{\circ}\right)$ at the South Galactic Cap, $|b|>45^{\circ}$. Since star counts at high Galactic latitudes are not strongly related to the radial distribution, they are well suited to study the vertical distribution of the Galaxy. The SCUSS magnitude $u$ and SDSS magnitudes (Juric et al. 2008) $g, r, i$, and $z$ were used for a total number of 4039908 stars in 18 fields (320 deg ${ }^{2}$ in total). Here, our work is restricted to the magnitude range $15<g<23$ for the study of the Galactic model parameters. The adopted model of stellar density distribution in this report includes only two disks (thin disk and thick disk) and a halo. We have chosen to use an exponential functional form for the disk and a de Vaucouleurs law for the halo. We try to derive the structural parameters (e.g. scale height) of the thin and thick disk populations using our data set. For this we calculate the stellar space density as a function of distance from the Galactic plane.

From the $\chi^{2}$ fit to the direct measurement of the stellar density distribution, we determine that the range of scale height for the thin disk varies from 180 to $250 \mathrm{pc}$. Although $180 \mathrm{pc}$ seems an extreme value, it is close to the lower limit in the literature. The range of scale height for the thick disk is from 400 to $800 \mathrm{pc}$, and the corresponding space number density normalization is $14.0-5.0 \%$ of the thin disk. Our results show that the scale height is variable with the observation direction, which cannot be attributed to statistical errors. The main reason can possibly be attributed to the disk (mainly the 
thick disk) being flared, with a scale height that increases with radius. This is consistent with the merger origin for the thick disk formation (Du et al. 2003, 2006, 2008). For the halo, we derive an estimate of its large-scale flattening and find that the axial ratio towards the Galactic center is more flatter $(\sim 0.4)$, while the shape of the halo in the anticentre direction is rounder with $c / a>0.4$. These results prove that the axial ratio of the halo may vary with distance and observation direction. It reflects the shape of the inner halo. In a word, the star counts for different lines of sight can be used directly to obtain a rough estimate of the shape of the stellar halo. Our solutions support the Galactic models with a flattened inner halo, possibly formed in part through a merger early in the Galaxy's history.

\section{References}

Du, C. H., Zhou, X., Ma, J., et al. 2003, A\& A, 407, 541

Du, C. H., Ma, J., Wu, Z. Y., \& Zhou, X. 2006, MNRAS, 72, 1304

Du, C. H., Wu, Z. Y., Ma, J., \& Zhou, X. 2008, ChJAA,5,566

Juric, M., Ivezic, Z., Brooks, A., et al. 2008, ApJ, 673, 864 